\title{
DISEÑO DE RECURSOS DIGITALES PARA ENTORNOS DE E-LEARNING EN LA ENSEÑANZA UNIVERSITARIA
}

\author{
(DESIGN OF DIGITAL RESOURCES FOR E-LEARNING ENVIRONMENTS AT THE UNIVERSITY)
}

\author{
María del Mar Sánchez Vera \\ Universidad de Murcia (España)
}

\section{RESUMEN}

La diversidad de tecnologías y recursos implican que al ciudadano del siglo XXI se le plantee una gran variedad de opciones para aprender a través de las tecnologías. Términos como e-learning y blended-learning no son desconocidos para los ciudadanos. Sin embargo, tenemos que tener en cuenta que la innovación tecnológica que implican estas nuevas vías de aprendizaje deben implicar una adecuada innovación pedagógica y metodológica. La creación de contenidos y recursos digitales para estos entornos no puede quedar al margen de los patrones pedagógicos necesarios para fomentar un proceso de enseñanza-aprendizaje adecuado. En este artículo se presenta un repaso por las distintas tipologías de aprendizaje que se pueden dar en la red y se exponen una serie de ideas y propuestas interesantes para el diseño e implementación de materiales digitales en la enseñanza universitaria, en donde, las herramientas Web 2.o y las nuevas tendencias, como los objetos de aprendizaje, no pueden pasar desapercibidos.

Palabras clave: e-Learning, blended learning, diseño, recursos, universidad.

\begin{abstract}
The different kinds of technology and resources that we can find nowadays shows that the citizens of the twenty-first century are faced with a variety of options for learning through technology. Words such as e-learning and blended learning are not unknown to the public. However, we must take into consideration that technological innovation needs to be supported by appropriate pedagogical and methodological innovation. The creation of digital content and resources for these environments cannot be left out of the pedagogical planning needed to promote the teaching-learning process in the right way. This article presents a review of different types of learning that can occur in the network and presents a number of interesting ideas and proposals for the design and implementation of digital materials in university teaching where the Web 2.0 tools and new trends, such as learning objects, cannot be avoided.
\end{abstract}

Keywords: e-Learning, blended learning, design, resource, university. 


\section{EDUCACIÓN A DISTANCIA, E-LEARNING Y BLENDED-LEARNING}

Encontramos un gran número de términos para caracterizar las nuevas formas de enseñanza-aprendizaje que han proporcionado las Tecnologías de la Información y la Comunicación (TIC). Podemos hablar de eLearning (electronic learning), m-learning (mobile learning), w-learning (wireless learning), b-learning (Blended Learning). Vamos a definir brevemente cada uno de ellos para poder adentrarnos con posterioridad en el diseño de recursos para estos entornos.

\section{La educación a distancia}

¿Cuándo comenzaríamos a hablar de educación a distancia? Posiblemente tendríamos que remontarnos a las antiguas civilizaciones como la egipcia y la griega en las que ya se utilizaban las cartas. Sin embargo, no nos vamos a extender en sus orígenes, sino en su concepto y, sobretodo, en las connotaciones que conlleva hablar de educación a distancia en la actualidad del mundo polisémico en el que nos movemos.

Encontramos diversas definiciones de educación a distancia. Para García Aretio $(1987,2000)$ la educación a distancia es un sistema de comunicación bidireccional que sustituye la interacción personal en el aula de profesor y alumno, por la acción sistemática y conjunta de diversos recursos didácticos, y el apoyo de una acción tutorial que propicia el aprendizaje autónomo del alumno. Este mismo autor en 2002 indica que, aunque la base de la definición de la educación a distancia está en sí misma al aportar el concepto de distancia, sí es cierto que podemos profundizar en algunos aspectos. De hecho, desde la pedagogía, los últimos estudios sobre la educación a distancia tratan temas como la presencia social del alumno o las modalidades de interacción que se encuentran en el proceso.

Caladine (2008) recoge los trabajos de Nipper (1989) y Reiser (2002), y otros destacados expertos en educación a distancia, y a partir de sus reflexiones este autor nos habla de seis generaciones en la educación a distancia. Al mismo tiempo que vamos a ir comprendiendo las distintas generaciones, vamos a poder comprobar el papel que la tecnología ha ido teniendo en la evolución de la educación a distancia:

- La primera generación es la que denomina el modelo por correspondencia. Es el que se realiza vía postal. 
- La segunda generación es la del modelo multimedia, que se caracteriza por la evolución que produjo los conceptos de hipermedia y multimedia a la hora de crear cursos a distancia. Ejemplo de esta generación es el vídeo.

- La tercera generación es la de la combinación de herramientas y telecomunicaciones, denominado el modelo de telelearning por Fozdar y Kuman (2007), en la cual se hace uso de una gran cantidad de nuevos medios, y tomando ante todo, las ventajas referidas a la evolución de las telecomunicaciones. Ejemplo de esta generación es la videoconferencia. En esta generación es en la que podemos percibir la separación entre dos tipos de tecnologías: la utilizada para proveer materiales, y la utilizada para mejorar la interacción y la comunicación entre personas. Es también en este momento cuando los conceptos de educación abierta y enseñanza flexible comienzan a sonar en los textos de los autores de la época.

- La cuarta generación es denominada por Taylor (1995) el modelo de aprendizaje flexible. Destaca este autor el incremento de experiencias universitarias que se produjo durante los 90 sobre cursos en línea, de modo que se refuerza la conexión entre la enseñanza a distancia y los modelos de aprendizaje flexible, que se caracterizan por dar aún mayor libertad a la flexibilidad de tiempo y lugar. Esta generación hace referencia a la aplicación de distintas técnicas de trabajo a través de TIC en la educación a distancia.

- La quinta generación es construida por Taylor (2001) sobre la cuarta, con la adición de ser una generación del Campus Virtual institucional de procesos y recursos, caracterizada por los sistemas de automatización que han incorporado los centros educativos. El autor lo denomina como un cambio en el rol de la tecnología en la educación superior porque llega un momento en el que los cursos en línea no suponen una experiencia ocasional sino que comienzan a formar parte del entramado de las instituciones. Los portales ofrecen a los estudiantes con un punto de acceso eficiente a los materiales y recursos didácticos, así como registros de su progreso y las comunicaciones. Esta generación es denominada por Fozdar y Kuman (2007) como el modelo de inteligencia flexible.

- La sexta generación incluida por Caladine (2008) es la Web 2.o. Lo podríamos considerar un modelo de avance del entorno interactivo. Esta autora afirma que cuando Taylors, en 2001, incluyó la quinta generación, los campos virtuales eran los protagonistas en la educación a distancia, sin embargo, desde entonces, la tecnología ha cambiado, hemos visto un incremento espectacular de las 
herramientas de Software Social. La Web 2.0 ha hecho que pasemos de una Web estática a una Web dinámica, que se caracteriza por la participación y la colaboración mediante redes sociales en Internet. Este movimiento remodelador de la Web ha dado lugar a algunas herramientas que se sirven del mismo, y en las que subyace la filosofía de colaboración que puede ser similar a la que se desarrolla en el Software libre, Wiki, youtube, Weblog, postcast, slide, etc... Entre ellas, encontramos las wikis y los Weblogs como las más extendidas. Participar en estas herramientas supone intercambiar pensamientos, crear planes, ubicar lugares, construir conceptos, en definitiva, compartir información. Las redes sociales son una realidad en Internet. Las redes son formas de interacción social, que se producen debido al intercambio que se produce de información, recursos... Normalmente se desarrollan a través de herramientas colaborativas, espacios que promueven el diálogo y la coordinación. La red de redes ha contribuido a que se elimine el espacio que separa a las personas y ha promovido este tipo de relaciones sociales. Este hecho es lo suficientemente significante para poder hablar de otra generación en la educación a distancia que es la que hace uso de las redes sociales, la que se caracteriza por los entornos virtuales que desarrolla el alumno y no únicamente el entorno institucional.

\begin{tabular}{|c|c|c|}
\hline Generación & Modelo & Algunos recursos \\
\hline 1 generación & Modelo de correspondencia & Materiales impresos \\
\hline 2 generación & Modelo multimedia & $\begin{array}{l}\text { Materiales impresos, cintas } \\
\text { de audio, vídeo }\end{array}$ \\
\hline 3 generación & Modelo de telelearning & $\begin{array}{l}\text { Comunicación mediante } \\
\text { satélite, videoconferencia, } \\
\text { broadcast }\end{array}$ \\
\hline 4 generación & $\begin{array}{l}\text { Modelo de aprendizaje } \\
\text { flexible }\end{array}$ & $\begin{array}{l}\text { Multimedia interactiva, } \\
\text { internet de www, } \\
\text { comunicación online }\end{array}$ \\
\hline 5 generación & $\begin{array}{l}\text { Modelo de inteligencia } \\
\text { flexible }\end{array}$ & $\begin{array}{l}\text { comunicación on-line, } \\
\text { campus virtuales, portales } \\
\text { institucionales }\end{array}$ \\
\hline 6 generación & $\begin{array}{l}\text { Modelo de avance del } \\
\text { entorno interactivo }\end{array}$ & $\begin{array}{l}\text { Web 2.o, Software social } \\
\text { (wikis, blogs), entornos } \\
\text { personalizados }\end{array}$ \\
\hline
\end{tabular}

Figura 1. Generaciones en la educación a distancia. A partir de Taylor (1995, 2001), Fozdar y Kumar (2007) y Caladine (2008) 
Este recorrido por las generaciones de la educación a distancia comenzó desde una perspectiva orientada a los materiales, y finalmente muy focalizada en el caso de la enseñanza superior. Obviamente, uno no supone una superposición sobre el otro, por ejemplo, aunque los materiales impresos se identifican fundamentalmente con la primera y la segunda generación, eso no quiere decir que en la actualidad no los sigamos utilizando.

A continuación analizaremos las modalidades de educación a distancia apoyadas en TIC, concretamente el eLearning y el Blended Learning.

\section{La educación a distancia a través de las TIC. E-Learning y Blended Learning}

Como afirma De Pablos (2004, p. 121), "la enseñanza no presencial puede ser más rentable que la presencial”, pero ¿qué es el eLearning? Las primeras ideas y experiencias con eLearning (telenseñanza en castellano) comienzan durante los 90 al mismo tiempo que la red evoluciona y se hace más rápida y accesible. En 1991, Benedikt publica un libro en el que lanza las bases teóricas de la arquitectura ciberespacial; este autor define el ciberespacio como una red multidimensional global soportada por los ordenadores.

Sobre eLearning encontramos una gran cantidad de definiciones (Bates, 1995; Rosenberg, 2001; Snajder, Verlic, Povalej y Debecv, 2007; Baleo, 2009). Ante la gran variedad de definiciones que se encuentran, vamos a indicar los aspectos más relevantes que son comunes a la mayoría de ellas:

- La educación on-line es educación a distancia: el eLearning puede realizarse en cualquier lugar y en cualquier tiempo.

- $\quad$ Se produce en la red.

- Los usuarios necesitan de ordenadores.

- Se amplían las perspectivas de aprendizaje dando un paso más allá de los paradigmas tradicionales de formación. 


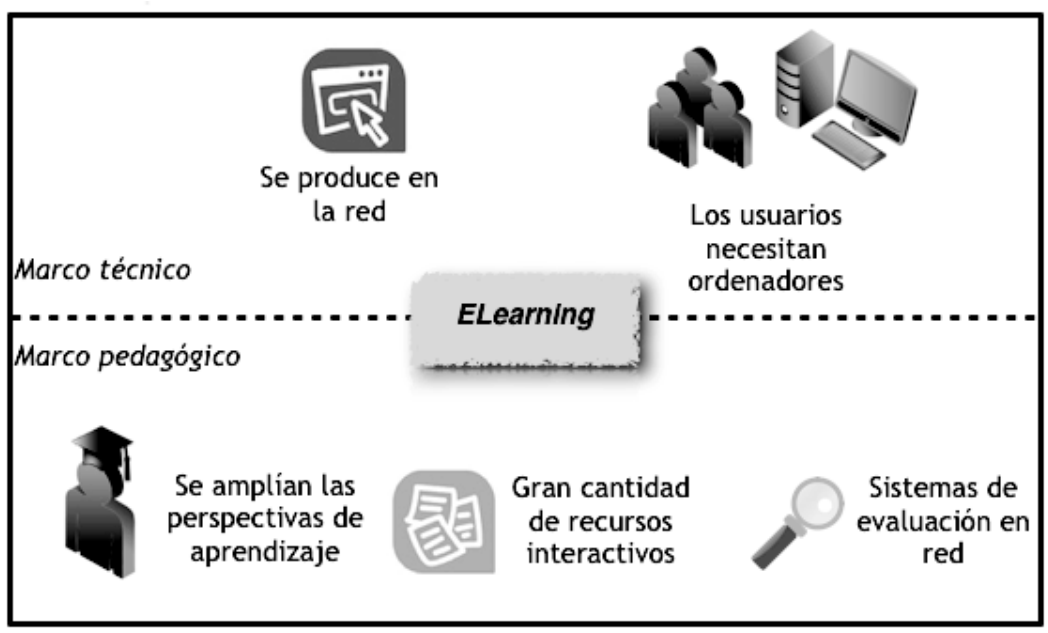

Figura 2. Algunos elementos que pueden caracterizar el eLearning

Al igual que sucede con la definición, encontramos también que son diversos los autores que incluyen las características principales que denotan este tipo de formación que se da a través de redes. Si nos centramos en conocer qué es y qué no es educación on-line podemos contemplar a Adell y Sales (1999), que nos indican claramente en su trabajo sobre el rol del profesor virtual varias premisas sobre qué es la educación on-line:

- La educación on-line es educación a distancia: el eLearning puede realizarse en cualquier lugar y en cualquier tiempo.

- La educación on-line es educación de adultos: en los cursos virtuales los alumnos adultos buscan la flexibilidad que les permite realizar la enseñanza a través de redes. A juicio de los autores, otra de las dimensiones de la educación en red es que los aprendices son normalmente adultos, como bien indican, formar adultos es muy diferente a formar niños o adolescentes.

- La educación on-line es comunicación mediada por el ordenador: es una de las características básicas que definen la educación en red, y es que la utilización de ordenadores es básica para la transferencia y almacenamiento de la información.

Actualmente las herramientas de la Web 2.0 están teniendo tanta importancia en los sistemas de aprendizaje en red que algunos autores (Downes, 2005; Caladine, 2008; Baelo, 2009) hablan de eLearning 2.0 como el proceso de enseñanza- 
aprendizaje que se realiza a través de las redes y que está caracterizado así mismo por el uso de herramientas de Software Social. En la actualidad seguimos utilizando los Campus Virtuales como sistema básico para desarrollar un curso de eLearning. Estos entornos se han ampliado para dar cabida a las herramientas de la Web 2.0, como puedan ser blogs y wikis, de modo que podemos aprovechar las ventajas del entorno ampliadas. El movimiento impulsor de Software libre y de licencias de acceso abierto al conocimiento no ha hecho sino ampliar estas perspectivas de aprendizaje. De hecho, el término eLearning 2.o supone una nueva visión sobre la Web. Este eLearning 2.o se diferencia del eLearning tradicional en la aplicación de las herramientas a los procesos de eLearning, afirma el autor que tiene su fundamentación pedagógica en el conectivismo de Siemens (2004), y se basa en (Baelo, 2009, p. 92) para caracterizarnos este concepto:

- El alumnado es el que crea y modifica los contenidos por medio de las herramientas Web 2.0.

- Las experiencias de aprendizaje se realizan gracias a la reutilización de contenidos de diversas fuentes.

- Se produce un cambio en el diseño e integración de las herramientas que permiten mayor flexibilidad y rapidez.

Pero el e-Learning no es el único tipo de educación que se produce a través de las TIC. En 2004, el profesor Bartolomé (2004) hablaba del fracaso del eLearning, haciendo referencia a que tras el entusiasmo inicial que suscitó el eLearning este no ha sabido responder a las expectativas que había creado. Este autor hace referencia a diferentes estudios que indican que se produjeron durante los años 2001 a 2003 paralizaciones de grandes proyectos y un parón en la inversión de infraestructuras. Sin embargo, reflexiona, no se puede hablar de fracaso del eLearning como sistema en sí mismo, sino del fracaso de algunas expectativas que se habían creado. Como indica Cabero (2006), una de las causas puede ser el tecnocentrismo, es decir, el situar a la tecnología por encima de la pedagogía, olvidando todas las variables didácticas que hay que tener en cuenta a la hora de la enseñanza en entornos virtuales. En aquel momento el Blended Learning surge como un elemento emergente a tener en cuenta. En 2003, la Sociedad Americana para el Aprendizaje y el Desarrollo identificó el Blended Learning como una de las tendencias emergentes en la sociedad del conocimiento. 
La palabra inglesa blend significa "combinación, mezcla", lo cual nos hace ya intuir qué puede significar el concepto de Blended Learning. Una de las definiciones más actualizadas nos las ofrece Llorente (2009, p. 19) que indica que "el Blended Learning combina la eficacia y la eficiencia de la clase presencial con la flexibilidad del eLearning". El Blended Learning supone una combinación de diferentes entornos, métodos de enseñanza y estilos de aprendizaje, por lo que debemos acercarnos más a la idea de concebir el Blended Learning como un entorno con grandes posibilidades educativas y comunicativas; por tanto, el Blended Learning sería "el aprendizaje que se caracteriza por la eficaz combinación de diferentes modalidades de aplicación, modelos de enseñanza y estilos de aprendizaje, y que se basa en una comunicación transparente entre todas las partes implicadas en un curso" (Heinze y Porcter, 2004, p. 19).

Desde lo presencial a lo virtual se pueden encontrar diversos modelos docentes en función del grado de interacción de las TIC (figura 3). Una integración curricular de la red, de forma más o menos significativa nos hace hablar de diferentes modelos de lo que se entiende por Blended Learning, lo que habitualmente podemos conocer como enseñanza semipresencial. También permite hablar de una flexibilización de los modelos tradicionales de enseñanza a distancia.

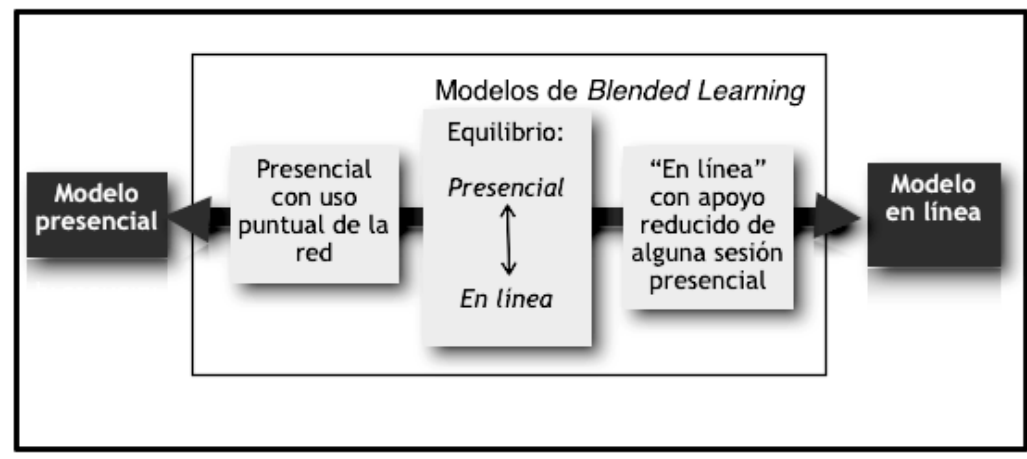

Figura 3. Modelos de Blended Learning y telenseñanza (Prendes, 2007)

\section{Entornos para el eLearning y el Blended Learning: Campus Virtuales y entornos personales de aprendizaje}

Los primeros centros educativos que se desarrollan totalmente a través de Internet son las denominadas por Hanna (2002) "universidades virtuales". Las instituciones de educación superior suelen utilizar plataformas de Campus Virtual 
para desarrollar los cursos virtuales. Una plataforma de eLearning está constituida por tres elementos funcionales:

- $\quad$ LMS (Learning Management System): es el punto de contacto entre los usuarios de la plataforma (profesores y estudiantes, fundamentalmente). Se encarga, entre otras cosas, de presentar los cursos a los usuarios, del seguimiento de la actividad del alumno, etc.

- $\quad$ LCMS (Learning Content Management System): engloba aspectos directamente relacionados con la gestión de contenidos y la publicación de los mismos. También incluye la herramienta de autor empleada en la generación de los contenidos de los cursos.

- Herramientas de comunicación: puesto que la comunicación entre el profesor y el estudiante pasa a ser virtual, deben proporcionarse los mecanismos necesarios para ello. Dentro de este grupo se incluyen chat, foros, correo electrónico, intercambio de ficheros, etc.

Podríamos definir el Campus Virtual como un entorno que utiliza las TIC para posibilitar la interacción entre profesores y alumnos.

Hay otro concepto que se ha desarrollado durante los últimos años cuando se habla de entornos virtuales de aprendizaje (que no de Campus Virtuales). Podemos pensar en cómo la idea de los entornos personales de aprendizaje puede estar transformando el eLearning. Hablar de PLE (Personal Learning Enviroment), en español EPA (Entorno Personal de Aprendizaje), es hacer referencia al "entorno de aprendizaje que desarrolla el alumno por sí solo en su proceso, que puede ser el que ofrece la institución, pero al mismo tiempo utiliza otras aplicaciones de la Web 2.o construyendo así su propia versión de un entorno de aprendizaje, su Entorno Personal de Aprendizaje” (Castañeda y Sánchez Vera, 2009, p. 178; Horton, 2001).

Un entorno personal de aprendizaje quedaría configurado por las herramientas virtuales que el alumno utiliza en su proceso de aprendizaje. Podemos encontrar, por ejemplo, un alumno que se mueva solo dentro del Campus Virtual de la asignatura, o por otro lado, un alumno que utilice blogs, wikis, u otro tipo de herramientas, en el desarrollo de la asignatura. El EPA estaría configurado, en ambos casos, por las herramientas que el alumno ha utilizado, que pueden provenir de la institución o pueden provenir de la espontaneidad del alumno al buscar más información por los canales que hoy ofrece la Web 2.0. Si el Campus Virtual ubica un lugar "no físico" en 
el ciberespacio, un EPA estaría presente en muchos "lugares", por decirlo de algún modo, el EPA lo conformarían todas las aplicaciones y espacios que el estudiante ha utilizado en el curso virtual.

\section{Elearning y Blended Learning en la Universidad}

En un análisis que la CRUE (2008) realizó sobre la situación de las TIC en la enseñanza universitaria en el contexto europeo y español, se indica que la presencia de las TIC en las universidades ha experimentado, por un lado, un incremento espectacular, aunque por el otro esta incorporación se ha realizado bajo los parámetros clásicos de entornos de docencia unilateral, por lo que en los próximos años será necesario rediseñar los métodos docentes para una integración efectiva de las TIC como herramientas interactivas.

En la Unión Europea, tanto en el Espacio Europeo de Educación Superior, como en otros proyectos de la comunidad, las TIC suponen una apuesta segura. El programa eLearning, por ejemplo, proponía en 2003 el fomento, divulgación y aprovechamiento de los resultados y de las buenas prácticas obtenidas de la aplicación de las TIC. El Plan i2010 indica en su página Web que se encarga de promover la contribución positiva que las Tecnologías de Información y la Comunicación pueden aportar a la economía, la sociedad y la calidad de vida personal. Entre las acciones llevadas a cabo en el año 2008-2009 por el plan i2010 se destacan:

- Proponer mejoras al sistema de normalización de las TIC de la UE.

- Adoptar un plan de acción para promover aún más la firma y autenticación electrónica.

- Aplicar el marco europeo para la facturación electrónica.

- Poner en marcha las iniciativas tecnológicas conjuntas como la primera verdadera Europa público-privado, las asociaciones de investigación.

- Promover las Plataformas Tecnológicas Europeas, en particular una cooperación más estrecha entre ellas.

- Promover el papel del sector público como primer comprador de la innovación. 
- Iniciar un proceso para garantizar el liderazgo europeo en las TIC con una comunicación sobre la investigación de TIC e Innovación.

- Promover el papel de las infraestructuras electrónicas de investigación en un entorno cambiante y global.

Sangrá (2001) nos indica que el término universidad virtual surgió como algo que debería englobar un concepto sistémico de la universidad ofrecida a los estudiantes y a la comunidad docente e investigadora, un concepto integral, de universidad en sí misma, suficientemente distinto del término Campus Virtual. Hanna (1998) explica que podemos encontrar distintos tipos de universidades en función del modelo de la incorporación quehace unainstitución deeducación superior delas TIC: universidades de educación a distancia basadas en la tecnología, instituciones privadas dirigidas a la enseñanza de adultos, universidades corporativas, alianzas estratégicas universidadempresa, organizaciones de control de acreditación y certificación, universidades tradicionales extendidas, y universidades multinacionales globales. El uso de las TIC en la enseñanza superior supone, sin duda, una enorme apuesta por la innovación de las metodologías, estrategias y modelos didácticos, pero su integración curricular en la enseñanza universitaria debe estar fundamentada en una reflexión profunda sobre los roles que deben adoptar los profesores en el proceso de enseñanza-aprendizaje, y sobre las ventajas que estas aportan al mismo. Ello supone que las instituciones universitarias deben realizar una apuesta potente para su uso, y esto implica (Solano, 2008, p. 129):

- Crear o ampliar la infraestructura de red, informática y audiovisual necesaria, para lo cual se requerirá un apoyo económico considerable que garantice la efectividad de los cambios introducidos.

- Adquirir las aplicaciones y equipamiento que contribuyan a los objetivos propuestos.

- Valorar la pertinencia de promover el uso de Software libre que permita la interoperatibilidad entre equipos e instituciones y contribuya a la democratización del conocimiento.

- Proveer la formación del profesorado en el uso de técnicas y estrategias didácticas basadas en el uso de las TIC, así como en el manejo de plataformas de telenseñanza, utilizadas bien para apoyo y complemento a la enseñanza presencial, bien para el desarrollo de cursos semipresenciales y no presenciales. 
- Propiciar la elaboración de planificaciones de situaciones de telenseñanza o semiprensenciales acordes con las normativas e imposiciones administrativas establecidas por la institución.

- Favorecer la cooperación y el intercambio de experiencias entre los alumnos, docentes, investigadores y personal de la administración.

- Promover el apoyo institucional para la creación de nuevos escenarios de aprendizaje apoyados en herramientas telemáticas y estrategias para la colaboración y la comunicación de docentes, alumnos, agentes externos y el resto de miembros de la comunidad universitaria.

\section{Diseño e implementación de recursos TIC para entornos en red}

Los materiales que se diseñan y utilizan en entornos TIC tienen unas diferencias a considerar respecto a lo que supone un formato de texto tradicional:

\begin{tabular}{|l|l|}
\hline \multicolumn{1}{|c|}{ Texto tradicional } & Materiales electrónicos interactivos \\
\hline Soporte papel & Soporte óptico y electrónico \\
\hline Comunicación de información secuencial & Informaciones no secuenciales \\
\hline $\begin{array}{l}\text { Determinación de una lectura obligada y } \\
\text { homogénea }\end{array}$ & $\begin{array}{l}\text { Flexibilidad en el recorrido por la } \\
\text { información }\end{array}$ \\
\hline $\begin{array}{l}\text { Mayor peso de la estructura lógica de la } \\
\text { argumentación (coherencia) }\end{array}$ & $\begin{array}{l}\text { Importancia de la estructura asociativa del } \\
\text { pensamiento (divergencia) }\end{array}$ \\
\hline $\begin{array}{l}\text { El orden de presentación de información } \\
\text { nos lleva a los objetivos de aprendizaje }\end{array}$ & $\begin{array}{l}\text { Posible utilización de material con distintos } \\
\text { fines y objetivos }\end{array}$ \\
\hline $\begin{array}{l}\text { El texto no puede ser modificado } \\
\text { Rigidez en la estructura de acceso a la } \\
\text { información }\end{array}$ & $\begin{array}{l}\text { El lector puede añadir nexos y otros textos } \\
\text { (coautor) }\end{array}$ \\
\hline Información textual e icónica estática & Información multicódigo dinámica dex de los to \\
\hline Limitación en la cantidad de información & Gran cantidad de información para navegar \\
\hline Difícil y cara actualización informativa & $\begin{array}{l}\text { Actualización permanente dela información } \\
\text { de forma sencilla y dinámica }\end{array}$ \\
\hline
\end{tabular}




\begin{tabular}{|l|ll|}
\hline \multicolumn{1}{|c|}{ Texto tradicional } & Materiales electrónicos interactivos \\
\hline $\begin{array}{l}\text { Las decisiones sobre la elaboración de } \\
\text { información son tomadas por el autor }\end{array}$ & $\begin{array}{l}\text { Implicación del lector en la toma de } \\
\text { decisiones sobre lo que quiere aprender }\end{array}$ \\
\hline $\begin{array}{l}\text { La información posee una organización } \\
\text { intrínseca determinada por el autor }\end{array}$ & $\begin{array}{l}\text { Mayor esfuerzo metacognitivo para } \\
\text { organizar la información }\end{array}$ \\
\hline $\begin{array}{l}\text { El texto orienta al lector sobrela información } \\
\text { relevante }\end{array}$ & $\begin{array}{l}\text { Posibilidad de desorientación en la } \\
\text { búsqueda de información relevante }\end{array}$ \\
\hline $\begin{array}{l}\text { El autor puede asumir que la lectura del } \\
\text { texto se hará siguiendo el orden secuencial }\end{array}$ & $\begin{array}{l}\text { El autor debe prever en su diseño diferentes } \\
\text { vías de exploración }\end{array}$ \\
\hline $\begin{array}{l}\text { Facilita tener una representación mental } \\
\text { global del contenido estudiado }\end{array}$ & $\begin{array}{l}\text { Dificultad para hacerse una representación } \\
\text { mental global del contenido }\end{array}$ \\
\hline $\begin{array}{l}\text { Limitaciones en la búsqueda y gestión de la } \\
\text { información }\end{array}$ & Gestión fácil y rápida de la información \\
\hline
\end{tabular}

Tabla 1. Diferencias entre el texto tradicional y los materiales electrónicos interactivos (Area y García Valcarcel, 2001, p. 418)

Ya sea para incorporar estos materiales interactivos en el aula presencial o para trabajar con ellos en un curso en red, vamos a hacer uso de diferentes recursos TIC, adaptando los que ya existen y creando otros nuevos.

Si queremos implementar en la enseñanza un recurso TIC, debemos de contemplar un proceso de integración curricular (Barroso, 2006, p. 23):

- Fase 1. Primer nivel de concreción: se refiere al centro, en esta fase deberíamos ir considerando los aspectos relacionados con el plan de integración de las TIC, que se referiría a la estructura organizativa del centro (plan de integración curricular, profesorado, distribución horaria...), y a las teorías de aprendizaje que sustentan la aplicación de las TIC en la escuela.

- Fase 2. Segundo nivel de concreción: hablamos aquí del nivel y/o ciclo educativo, en donde los diferentes equipos tendrían que reunirse para realizar las actuaciones pertinentes en la programación, concretando los objetivos que se modificarán, los nuevos contenidos a introducir; es decir, establecer acciones coordinadas entre todo el profesorado del ciclo. 
- Fase 3. Tercer nivel de concreción: hace referencia a las actividades a realizar que nos posibiliten la adquisición de nuevos aprendizajes, reforzar aprendizajes, búsqueda de información, fomentar la creatividad, utilizar las TIC como medio de expresión.

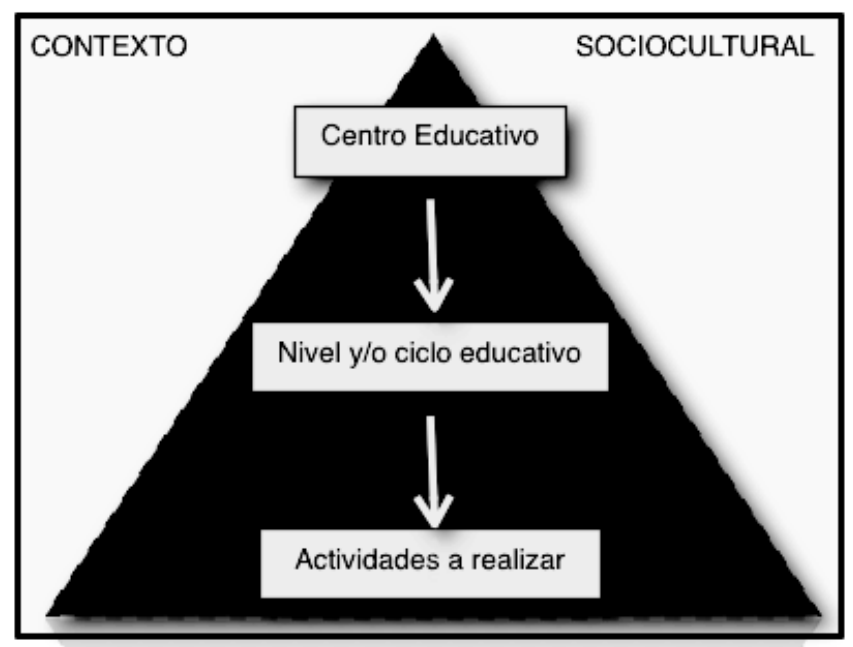

Figura 4. Fases en el proceso de integración curricular de las TIC. A partir de Barroso (2006)

Estas fases nos hacen comprender que las TIC no pueden ser impuestas a la ligera a un grupo de estudiantes; su implementación requiere un proceso de reflexión y planificación adecuado, que ha de terminar en una evaluación igualmente planificada en la organización previa y que nos sirva para mejorar los futuros procesos.

Desde el principio, muchos esfuerzos en la creación de programas informáticos se han encaminado hacia la perspectiva educativa. Marqués (1996) considera la definición de Software educativo, programas educativos y programas didácticos como términos sinónimos para designar a los programas de ordenador creados con la finalidad de ser utilizados para facilitar los procesos de enseñanza y aprendizaje (medios didácticos). El mismo autor señala, que siguiendo su propia definición, más basada en el criterio de finalidad que de funcionalidad, quedarían entonces descartados los programas empresariales, que también existen en los centros educativos con funciones didácticas, pero con fines lucrativos.

Los contenidos digitales didácticos son algo más que un medio que incorporamos a la enseñanza, ya que es un elemento creado específicamente para ello. Llorente (2006), en una propuesta de evaluación de este tipo de materiales digitales, hacía 
mella en una serie de variables que no se limitan al mero entorno informático del programa. Marqués estableció, ya en 1997, la idea de que los programas educativos comparten cinco características esenciales:

- Son materiales elaborados con una finalidad didáctica.

- Utilizan el ordenador como soporte en el que los alumnos realizan las actividades que ellos proponen.

- Son interactivos, es decir, contestan inmediatamente las acciones de los estudiantes y permiten un diálogo y un intercambio de informaciones entre el ordenador y los estudiantes.

- Individualizan el trabajo de los estudiantes, ya que se adaptan al ritmo de trabajo de cada uno y pueden adaptar sus actividades según las actuaciones de los alumnos.

- Son fáciles de usar. Los conocimientos informáticos necesarios para utilizar la mayoría de estos programas son similares a los conocimientos de electrónica necesarios para usar un vídeo, es decir, son mínimos, aunque cada programa tiene unas reglas de funcionamiento que es necesario conocer.

Quizás el Software educativo se preocupa más de precisar un tipo de alumno adaptado a la tecnología, y no una tecnología adaptada al alumno, o por lo menos esa debería de ser la premisa básica que habría que tener en cuenta al diseñarlo.

El Software, como elemento básico del funcionamiento de un ordenador, ha transformado y abierto nuevas posibilidades comunicativas, que se han visto incrementadas enormemente con la inclusión de Internet, y la aparición de nuevos programas no vienen sino a ampliar la oferta de recursos para la enseñanza. Durante estos últimos años, empresas, instituciones y particulares han desarrollado multitud de programas y que se han introducido en la escuela como innovaciones. Sin embargo, lo básico no está en la herramienta, sino en el método, es decir, lo importante no es lo innovadoramente tecnológico que sea el recurso, sino la estrategia pedagógica que se utiliza, por lo que, en cada situación específica habrá que decidir qué metodología es la más adecuada.

Como sabemos, el primer discurso que giraba en torno a la inclusión de los medios en la enseñanza se presentaba desde una perspectiva más teórico-instrumental, que 
fue superada por otra más curricular y didáctica, en la cual se entiende el medio como un elemento del currículum (Cabero, 2001). Cuando contemplamos el medio desde esta perspectiva curricular entendemos que el medio no funciona por sí mismo, de acuerdo con Cabero (1994), esta idea comenzó a consolidar una nueva perspectiva acerca de la concepción del uso de medios:

- Los medios no son otra cosa que unos elementos curriculares más.

- El aprendizaje no está en función del medio, sino del método y estrategia instruccional que apliquemos.

- $\quad$ El profesor es el elemento más significativo en la concreción del medio.

- Y que para innovar, cambiar y mejorar los centros no es suficiente con la incorporación de medios, por muy sofisticados que sean y por muchas posibilidades técnicas y expresivas que permitan.

Como se puede comprobar a partir de esta concepción, se considera al docente como agente principal impulsor en el uso de medios. Su rol es fundamental porque la última tecnología no tiene por qué ser la mejor, la estrategia instruccional del docente y el contexto son los factores determinantes para el uso del medio, es decir, hay que atender al proceso de enseñanza-aprendizaje, a los aspectos didácticos. Y es aquí en donde el docente juega un papel fundamental.

Siguiendo con las características de los materiales digitales, Cabero, Morales, Barroso, Román y Romero (2004), indican algunos criterios que tenemos que tener en cuenta a la hora de diseñar este tipo de materiales, que son:

- Autoridad científica tanto de la institución como de las personas que participan elaborando y proponiendo documentos.

- Calidad técnica de la página en lo que respecta a diseño gráfico.

- Velocidad de carga.

- Navegabilidad y facilidad y comprensibilidad del desplazamiento en los diferentes sitios y lugares. 
- Disposición de sistema de ayuda que auxilie al estudiante por los diferentes contenidos que se ofrecen y por las diferentes herramientas que se le presentan.

- Incorporación de un motor de búsqueda de contenidos.

- Comprensibilidad y adaptación de la información al usuario al cual va destinado.

- Veracidad y cientificidad de la información que se le presenta.

- Posibilidades de interactividad que permite.

Los criterios pedagógicos son importantes a la hora de diseñar un material educativo, tal y como indican muy acertadamente Adell y Sales (1999): "No se trata de sustituir al cartero para distribuir los materiales de estudio a una masa de estudiantes y al teléfono para la consulta ocasional de dudas y problemas en el estudio de los materiales [...]. La "perspectiva industrial" de producción en masa de productos idénticos y adoptar una visión más personalizada y artesanal de un proceso bastante más costoso de lo que se asume habitualmente. Si no es así, nos encontraremos con los mismos perros con distintos collares".

\section{Objetos de aprendizaje}

Una de los principales corrientes respecto al diseño de materiales educativos para espacios virtuales de aprendizaje es el movimiento de los objetos de aprendizaje. Los objetos de aprendizaje permiten el intercambio de contenidos educativos. Existen varias definiciones acerca de los objetos de aprendizaje. Wiley (2006) define el objeto de aprendizaje como "un recurso digital que puede ser reutilizado para facilitar el aprendizaje". Varas (2003) amplía la definición entendiendo que los objetos de aprendizaje son piezas individuales autocontenidas y reutilizables de contenido que sirven a fines instruccionales. Esta misma autora nos indica que los componentes pedagógicos básicos de un objeto de aprendizaje son: objetivos, contenido, actividad de aprendizaje y evaluación. 


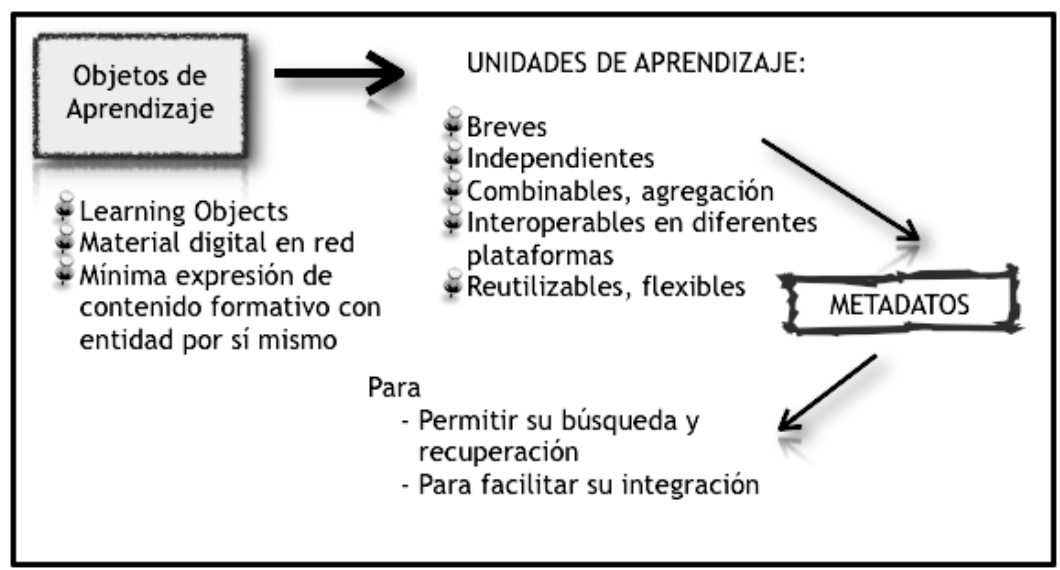

Figura 5. Concepto de objeto de aprendizaje a partir de Castañeda, López Vicent y Sánchez Vera (2007)

Los objetos de aprendizaje deben estar albergados y organizados en metadatos (figura 5), de manera tal que el usuario pueda identificarlos, localizarlos y utilizarlos para propósitos educacionales en ambientes basados en Web, para lo cual es necesario concertar unos estándares. Los objetos de aprendizaje han de ser simples, compartidos y reutilizados, de ahí la gran importancia que tienen para la enseñanza, porque nos permiten obtener información útil para elaborar módulos educativos. Es conveniente indicar que información no es formación; los objetos de aprendizajes han de ser adaptados al contexto escolar (Martínez y Prendes, 2007).

Siendo una de las finalidades de los objetos de aprendizaje la reutilización, es necesario que existan unos estándares que permitan compartir estos recursos digitales. Una de las principales especificaciones de estandarización es SCORM (Sharable Content Object Reference Model). SCORM hace posible el crear contenidos que puedan importarse dentro de sistemas de gestión de aprendizaje diferentes, siempre que estos soporten la norma SCORM.

Los objetos de aprendizaje suponen una manera sencilla de crear materiales digitales, ya que no son cursos completos, sino unidades de contenido, de manera que existen muchas experiencias de repositorios de objetos de aprendizaje del profesorado universitario. En 2006, el estudio de la ARL mostró el incremento que existía de este tipo de repositorios en las instituciones universitarias, e indicó que el $78 \%$ de las 87 bibliotecas universitarias que participaban en la encuesta tenían un repositorio ya en marcha o lo tendría instalado entre 2006 y 2007. 


\section{REFERENCIASBIBLIOGRÁFICAS}

Adell, J.; Sales, M. A. (1999). El profesor online: elementos para la definición de un nuevo rol docente. En: Cabero, J. (Coord). EDUTEC. Nuevas Tecnologías en la formación flexible y a distancia. Secretariado de Recursos Audiovisuales y Nuevas Tecnologías. [en línea] Disponible en: http://tecnologiaedu. us.es/edutec/paginas/105.html (consulta 2011, 20 de marzo).

Area, M.; García Valcarcel, A. (2001). Los materiales didácticos en la era digital, del texto impreso a los Web inteligentes. En: Area, M. (Coord.). Educar en la Sociedad de la Información. (409-441). Bilbao: Descleé.

Baleo, R. (2009). El e-learning, una respuesta educativa a las demandas de las sociedades del siglo XXI. Pixelbit: Revista de Medios y Educación, 35, (87-96).

Bates, A. W. (1995). Technology, e-learning and distance education. Routledge: Oxon. [en línea] Disponible en: http:// books.google.es/books?hl=es\&lr=\&id $=\mathrm{yOpH} 1 \mathrm{aOuopcC} \&$ oi $=$ fnd\&pg $=$ PR6\& $\mathrm{dq}=$ assessment $+\mathrm{e}+$ learning\&ots $=\mathrm{juG}$ $\underline{6 Z \text {-j-Sl\&sig=f1BaNtgFG3VnVjorUse }}$ $\mathrm{z} 4 \mathrm{Ee} 8 \mathrm{xoM} \# \mathrm{v}=$ onepage $\& \mathrm{q}=$ assessme nt\%20e\%2olearning\&f=false (consulta 2011, 28 de enero).

Bartolomé, A. (2004). Blended Learning. Conceptos básicos. Pixel bit: Revista de Medios y Comunicación, 23, (7-20).

Barroso, J.; Romero, R. (2007). La informática, los multimedia y los hipertextos en la enseñanza. En: Cabero, J. (Coord.). Las Nuevas Tecnologías aplicadas a la educación. Madrid: McGraw Hill.

Cabero, J. (1994). Actitudes hacia los ordenadores y la informática. En: Cebrián de la Serna, M. (Dir.). Medios y recursos didácticos. (85-98).
Málaga: Servicio de Publicaciones de la Universidad de Málaga.

Cabero, J. (2001). Tecnología Educativa: diseño y utilización de medios en la enseñanza. Barcelona: Paidós, papeles de comunicación.

Cabero, J. (2006). Bases pedagógicas del e-learning. Revista de Universidad y Sociedad del Conocimiento, 3, (1). UOC. [en línea] Disponible en: http:// mundoacademico.unb.br/users/ ledafior/1111414851.pdf (consulta 2011, 20 de mayo).

Cabero, J.; Morales, J. A.; Barroso, J.; Román, P.; Romero, R. (2004). La red como instrumento de formación. Bases para el diseño de materiales didácticos. Pixelbit. Revista de Medios y Educación, 22, (5-23).

Caladine, R. (2008). Enhancing e-learning with media.rich content ans interactions. Hershey-New York: Information Science Publishing.

Castañeda, L.; Sánchez Vera, M. M. (2009). Entornosdee-learning paralaenseñanza superior: entre lo institucional y lo personalizado. Pixelbit: Revista de Medios y Educación, 35, (175-191).

CRUED (2008). Informe CRUE-TIC sobre las TIC en las Universidades. Madrid: CRUE. [en línea] Disponible en: http:// www.crue.org/prensa/2008octubre/ NP- UNIVERSITIC 2008.pdf (consulta 2011, 15 de Junio).

De Pablos, J. (2004). La formación superior y el reto de las Nuevas Tecnologías de la información. En: Martínez, F.; Prendes, M. P. Nuevas Tecnologías y educación. Madrid: Pearson.

Downes, S. (2005). E-learning 2.o. E-learn Magazine, 26.

Fozdar, B. I.; Kumar, L. S. (2007). Mobile learning and student retention. International Review of Research in 
Open and Distance Learning, 8 (2), (116).

García Aretio, L. (1987). Hacia una definición de Educación a Distancia. Boletín informativo de la Asociación Iberoamericana de Educación Superior a distancia, $\mathrm{n}^{\circ} .18$.

García Aretio, L. (2000). La educación a distancia. De la teoría a la práctica. Barcelona: Ariel.

Hanna, D. (1998). Higher education in an era of Digital Competition: emerging organizational models. Journal of Asynchonous Learning Networks, 2, (1).

Hanna, D. (2002). Las universidades de educación a distancia basadas en las tecnologías. En: Hanna, D. (Ed.). La enseñanza universitaria en la era digital. Octaedro. EUB. Barcelona.

Heinze, A.; Porcter, C. (2004). Reflections on the use of Blended Learning. Education in a Changing Enviroment. Universidad de Salford: Education Development Unit of Salford.

Llorente, M. C. (2009). Formación semipresencial apoyada en la red (Blended Learning). PsicoEduca. Madrid: Eduforma.

Marqués, P. (1996). El software educativo. Universidad Autónoma de Barcelona. [en línea] Disponible en: http:// www.lmi.ub.es/te/any96/marques software/ (consulta 2008, 12 de diciembre).

Martínez, F.; Prendes, M. P. (2007). Las TIC en el aula como elemento de la flexibilización docente. En: Martínez, F.; Prendes, M. P. (Coord.). La enseñanza con objetos de aprendizaje. Madrid: Dykinson.

Nipper, A. (1989). Third generation distance learning and compjuter conferencing. En: Mason, R.; Kaye, A. (Eds.). Mindweave, communication, computers and distance education. Oxford: Pergamon.
Prendes, M. P. (2007). Selección e integración de medios en la enseñanza. En: Cabero, J. (Coord). Tecnología Educativa. Madrid: McGraw Hill.

Rosenberg, M. J. (2001). E-learning. Strategies for Delivering Knowledge in the Digital Age. New York: McGrawHill.

Sangrá, A. (2001). La calidad en las experiencias virtuales de Educación Superior. Actas de la conferencia Internacional sobre Educación Superior, formación y Nuevas Tecnologías. (641-625). [en línea] Disponible en: http://reddigital. cnice.mec.es/6/Documentos/docs/ articulo13 material.pdf (consulta 2010, 22 de abril).

Siemens, G. (2004). Connectivism: a learning theory for the digital age. E-Learn Space. [en línea] Disponible en: http://www.elearnspace.org/ Articles/connectivism.htm (consulta 2010, 20 de febrero).

Solano, I. M. (2008). Nuevos modelos y nuevos medios para la colaboración en las universidades del siglo XXI. En: Martínez, F. (Coord.) Incorporación de las TIC en los programas académicos de las Universidades Estatales Costarricenses. (127-146). Murcia: Diego Marín.

Snajder, M.; Verlic, M.; Povalej, P.; Debevc, M. (2007). Pedagogical evaluation of e-learning courses. Adapted pedagogical index. Conference ICL2OO7. Austria: Villach.

Taylor, J. (1995). Distance education technologies: the fourth generation. Australian Journal of Educational Technology, 11, 2, (1-7).

Taylor, J. (2001). Fifth generation distance education. Higher Education Series, report n.4 o. Canberra, Australia: Department of Education, Training and Youth Affairs. 
Varas, L. M. (2003). Repositorio de Objetos de Aprendizaje. [en línea] Disponible en: http://www.alejandria.cl/recursos/ documentos/documento varas.doc (consulta 2011, 17 de junio de 2011).
Wiley, D. (2006). Guetting Axiomatic About Learning Objects: lanzando axiomas sobre objetos de aprendizaje. Laboratorio Virtual. Departamento de Lenguajes y Sistemas Informáticos. [en línea] Disponible en: http://www. reusability.org/axiomatic.pdf (consulta 2011, 17 de marzo).

\section{PERFIL ACADÉMICO Y PROFESIONAL DE LA AUTORA}

María del Mar Sánchez Vera. Doctora en Pedagogía por la Universidad de Murcia y miembro del Grupo de Investigación de Tecnología Educativa de la Universidad de Murcia. En su experiencia profesional se encuentra una beca de formación en tecnologías de la información del Centro de Información y Comunicación Educativa (CNICE) del Ministerio de Educación y una beca de investigación predoctoral FPU en el Departamento de Didáctica y Organización Escolar de la Universidad de Murcia. Ha realizado estancias de investigación en la Universidad de Cambridge y en la Universidad John Moore de Liverpool en Reino Unido. Su especialidad es la formación en la aplicación de las TIC en la enseñanza. Ha publicado artículos en revistas nacionales e internacionales, y ha participado en congresos y talleres relacionados con el uso de diversas herramientas Web 2.0 en la educación. Actualmente es docente de las asignaturas de "Planificación de la Acción Educativa" e "Investigación y TIC" en el grado de Magisterio de Educación Primaria en la escuela adscrita a la Universidad de Murcia, Departamento de Didáctica y Organización Escolar de la Facultad de Educación de la Universidad de Murcia.

E-mail: mmarsanchez@um.es

DIRECCIÓN DE LA AUTORA

María del Mar Sánchez Vera

Calle Picos de Urbión, $\mathrm{n}^{\circ} 10,1^{\circ} \mathrm{C}$.

CP: 30310. Cartagena, Murcia, España

Fecha de recepción del artículo: 29/08/11

Fecha de aceptación del artículo: 13/12/11 


\section{Como citar este artículo:}

Sánchez Vera, M. M. (2012). Diseño de recursos digitales para entornos de e-learning en la enseñanza universitaria. RIED. Revista Iberoamericana de Educación a Distancia, volumen 15, n 2, pp. 53-74. 